Article

\title{
The Sensitivity of Grating-Based SPR Sensors with Wavelength Interrogation
}

\author{
Jianjun Cao* $*$, Yuan Sun, Yan Kong and Weiying Qian \\ School of Science, Jiangnan University, Wuxi 214122, China; jndxsy1225@126.com (Y.S.); \\ ykong@jiangnan.edu.cn (Y.K.); wyqian@jiangnan.edu.cn (W.Q.) \\ * Correspondence: jianjuncao@jiangnan.edu.cn; Tel.: +86-1835-2553-583
}

Received: 10 December 2018; Accepted: 18 January 2019; Published: 19 January 2019

check for updates

\begin{abstract}
In this paper, we derive the analytical expression for the sensitivity of grating-based surface plasmon resonance (SPR) sensors working in wavelength interrogation. The theoretical analysis shows that the sensitivity increases with increasing wavelength and is saturated beyond a certain wavelength for $\mathrm{Au}$ and $\mathrm{Ag}$ gratings, while it is almost constant for Al gratings in the wavelength range of 500 to $1000 \mathrm{~nm}$. More importantly, the grating period $(P)$ and the diffraction order $(m)$ dominate the value of sensitivity. Higher sensitivity is possible for SPR sensors with a larger grating period and lower diffraction order. At long wavelengths, a simple expression of $P /|\mathrm{m}|$ can be used to estimate the sensor sensitivity. Moreover, we perform experimental measurements of the sensitivity of an SPR sensor based on an $\mathrm{Al}$ grating to confirm the theoretical calculations.
\end{abstract}

Keywords: surface plasmon resonance; metal grating; sensitivity enhancement; biosensor

\section{Introduction}

Biosensors based on surface plasmon resonance (SPR) have received much attention since their first application in gas sensing in 1983 [1]. The ability to detect very small changes in the refractive index makes SPR sensors a good platform to characterize gases, chemical molecules, and living cells, showing advantages of label-free and real-time detection [2-5]. Because the surface plasmon wave vector is larger than the free space one, excitation of SPR needs the help of high index optical elements like a prism [6,7], patterned nanostructures like diffraction grating [8,9], or optical fibers [10-15]. Nowadays, the prism-based SPR sensors are developed into commercial products due to their extremely high sensitivity. However, the use of prisms makes this design inevitably bulky. In recent years, there is growing interest in developing integrated SPR sensors to meet the requirements of portable point of care applications. Grating-based SPR sensors are suitable candidates for compact or integrated biosensors [16-21]. For example, an integrated chip with a gold grating substrate, which is only $1 \mathrm{~cm}^{2}$ in its size, is successfully fabricated [18]. However, the sensitivity of this type of SPR sensors has not been fully explored.

The sensitivity $(S)$ of a sensor is referred to by the ratio between the shift of the wavelength or angle and the shift of the refractive index. Theoretical analysis of the sensitivity is important for optimal design of sensors [22-28]. Previous literature has reported the dependence of sensitivity on the system parameters in different sensing methods. For prism-based SPR sensors in wavelength mode, $S$ increases as wavelength increases, and silver films have higher sensitivity than gold films $[29,30]$; for that in angular mode, $S$ is high at short wavelengths and tends to be constant at long wavelengths. For grating-based SPR sensors in angular mode, in the wavelength range of $600-1000 \mathrm{~nm}, S$ increases as wavelength increases and grating period decreases for the first diffraction order; for that in the wavelength mode, which is very close to the condition discussed in this paper. Homola et al. find that $S$ approaches $\lambda / n_{a}$ at long wavelengths, where $\lambda$ is the wavelength and $n_{a}$ is the refractive index of 
the analyte [30]. However, in their theoretical derivation the incident angle in the analyte was treated as a fixed value, which was hard to be realized in practical experiments. In practical experiments, the incident angle in air is fixed, while the angle in the analyte varies with its refractive index according to Snell's law.

In this paper, we derive the theoretical expression for the sensitivity of grating-based SPR sensors working in wavelength interrogation. The influences of system parameters, namely the operating wavelength, the grating period, the type of metal, and the refractive index of the analyte, on the sensitivity are comprehensively discussed. Furthermore, we perform experimental measurements of the sensitivity of a SPR sensor based on an Al grating to confirm our theoretical calculations.

\section{Theoretical Analysis}

The considered optical geometry of the SPR sensor is sketched in Figure 1. It consists of a metal grating and a fluidic channel. The metal grating has a sinusoidal profile with a period of $P$. The analyte filling in the fluidic channel has a flat top surface. To excite SPR at a given wavelength, a collimated white light with TM polarization (electric vector perpendicular to the grooves of the grating) is the incident on the sensor at a specific angle of $\theta$. The reflected light is then sent to a spectrometer that measures the reflection spectra. The excitation of SPR will be observed as an absorption dip in the spectra.

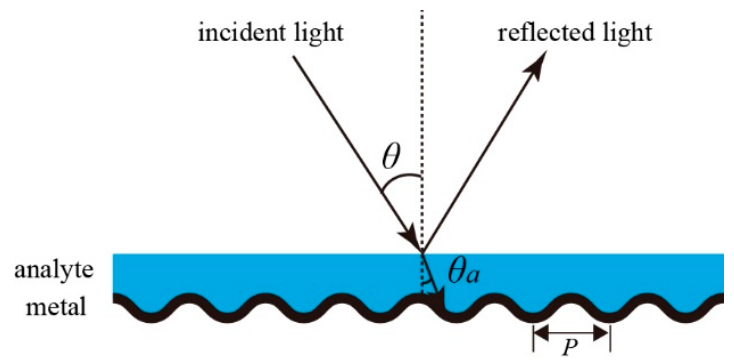

Figure 1. Schematic diagram of the grating-based surface plasmon resonance (SPR) sensor.

The key to excite SPR is wave vector matching of the incident light and the surface plasmon polariton. For a surface plasmon propagating at the interface between a metal layer with dielectric constant of $\varepsilon_{m}$ and a dielectric layer with dielectric constant of $\varepsilon_{d}$, its wave vector can be expressed as:

$$
k_{s p}=\frac{2 \pi}{\lambda} \sqrt{\frac{\varepsilon_{m} \varepsilon_{d}}{\varepsilon_{m}+\varepsilon_{d}}} .
$$

This wave vector is always greater than the in-plane wave vector of the incident light, which is $k_{x}=\frac{2 \pi}{\lambda} n_{a} \sin \theta_{a}\left(n_{a}\right.$ is the refractive index of the analyte and $\theta_{a}$ is the angle in the analyte). In grating coupling scheme, the wave vector of the diffracted beam $\left(k_{d}\right)$ is the sum of $k_{x}$ and the grating wave vector $[3,30]$ :

$$
k_{d}=\frac{2 \pi}{\lambda} n_{a} \sin \theta_{a}+m \frac{2 \pi}{P}
$$

where $m=0, \pm 1, \pm 2, \ldots$ is the diffraction order. Therefore, the SPR excitation condition is now $k_{d}=k_{s p}$, which can be written as:

$$
n_{a} \sin \theta_{a}+m \frac{\lambda}{P}= \pm \sqrt{\frac{\varepsilon_{m} n_{a}^{2}}{\varepsilon_{m}+n_{a}^{2}}}
$$

In this equation, $\varepsilon_{d}$ is replaced by $n_{a}^{2}$, the sign ' \pm ' on the right side corresponds to surface plasmons propagating along the positive or negative direction. In practical experiments, the incident angle in air 
( $\theta$ in Figure 1 ) is fixed, while $\theta_{a}$ varies with $n_{a}$ according to Snell's law, which is $\sin \theta=n_{a} \sin \theta_{a}$. Thus, Equation (3) should be modified as:

$$
\sin \theta+m \frac{\lambda}{P}= \pm \sqrt{\frac{\varepsilon_{m} n_{a}^{2}}{\varepsilon_{m}+n_{a}^{2}}}
$$

Here, we take particular concerns in the spectra range of 500 to $1000 \mathrm{~nm}$, where high-performance silicon detectors are applicable. Assuming that the material of the metal grating is Au and the refractive index of the analyte is 1.34, the incident angle to excite SPR is calculated as a function of the resonant wavelength for four different grating periods of $400 \mathrm{~nm}, 600 \mathrm{~nm}, 800 \mathrm{~nm}$, and $1000 \mathrm{~nm}$ for the first diffraction order. The results are shown in Figure 2. Generally, the angle decreases with increasing wavelength. Due to the constraint of $|\sin \theta| \leq 1$, SPR cannot be excited at long wavelengths for $P=400 \mathrm{~nm}$, as well as short wavelengths for $P=1000 \mathrm{~nm}$.

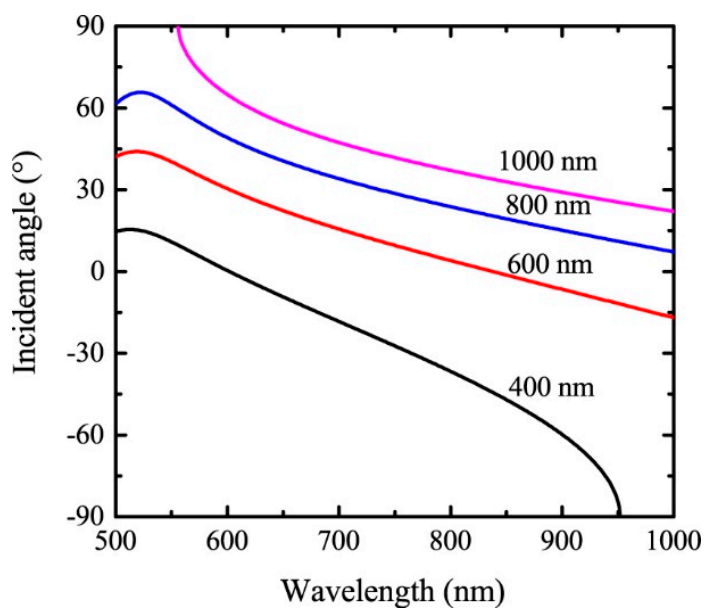

Figure 2. The angle of incidence as a function of the resonant wavelength for four different grating periods. The material of the grating is $\mathrm{Au}$ and $n_{a}=1.34, m=1$.

By differentiating Equation (4) in $\lambda$ and $n_{a}$, the analytical expression for the sensor sensitivity is obtained as:

$$
S=\frac{\mathrm{d} \lambda}{\mathrm{d} n_{a}}=\frac{\left(\frac{\varepsilon_{m}}{\varepsilon_{m}+n_{a}^{2}}\right)^{\frac{3}{2}}}{\frac{n_{a}^{3}}{P}-\frac{\mathrm{d} \mid}{2 \sqrt{\varepsilon_{m}}\left(\varepsilon_{m}+n_{a}^{2}\right)^{\frac{3}{2}}} \frac{\mathrm{d} \varepsilon_{m}}{\mathrm{~d} \lambda}} .
$$

For simplicity, we use the Drude model for the dielectric constant of metal to calculate $\frac{\mathrm{d} \varepsilon_{m}}{\mathrm{~d} \lambda}$. The Drude model is

$$
\varepsilon_{m}(\omega)=1-\frac{\Omega_{P}^{2}}{\omega\left(\omega-i \Gamma_{0}\right)},
$$

where $\omega=2 \pi c / \lambda, \Omega_{P}=\sqrt{f_{0}} \omega_{P}$ is the plasma frequency, $f_{0}$ is the oscillator strength, and $\Gamma_{0}$ is the damping constant. The values of $f_{0}, \omega_{P}$, and $\Gamma_{0}$ are obtained from [31,32]. Differentiating Equation (6) in $\lambda$, we obtain

$$
\frac{\mathrm{d} \varepsilon_{m}}{\mathrm{~d} \lambda}=\frac{\Omega_{P}^{2} \omega^{2}\left(i \Gamma_{0}-2 \omega\right)}{2 \pi c\left(\omega^{2}-i \Gamma_{0} \omega\right)^{2}}
$$

By substituting $\frac{\mathrm{d} \varepsilon_{m}}{\mathrm{~d} \lambda}$ using Equation (7) and $\varepsilon_{m}$ using Lorentz-Drude model into Equation (5), the value of the sensitivity can be calculated. Figure 3 shows the calculated sensor sensitivity versus 
the wavelength for grating periods of $400 \mathrm{~nm}, 600 \mathrm{~nm}, 800 \mathrm{~nm}$, and $1000 \mathrm{~nm}$ with system parameters of $n_{a}=1.34, m=1$. The materials for the gratings in Figure $3 \mathrm{a}-\mathrm{c}$ are $\mathrm{Au}, \mathrm{Ag}$, and $\mathrm{Al}$, respectively.
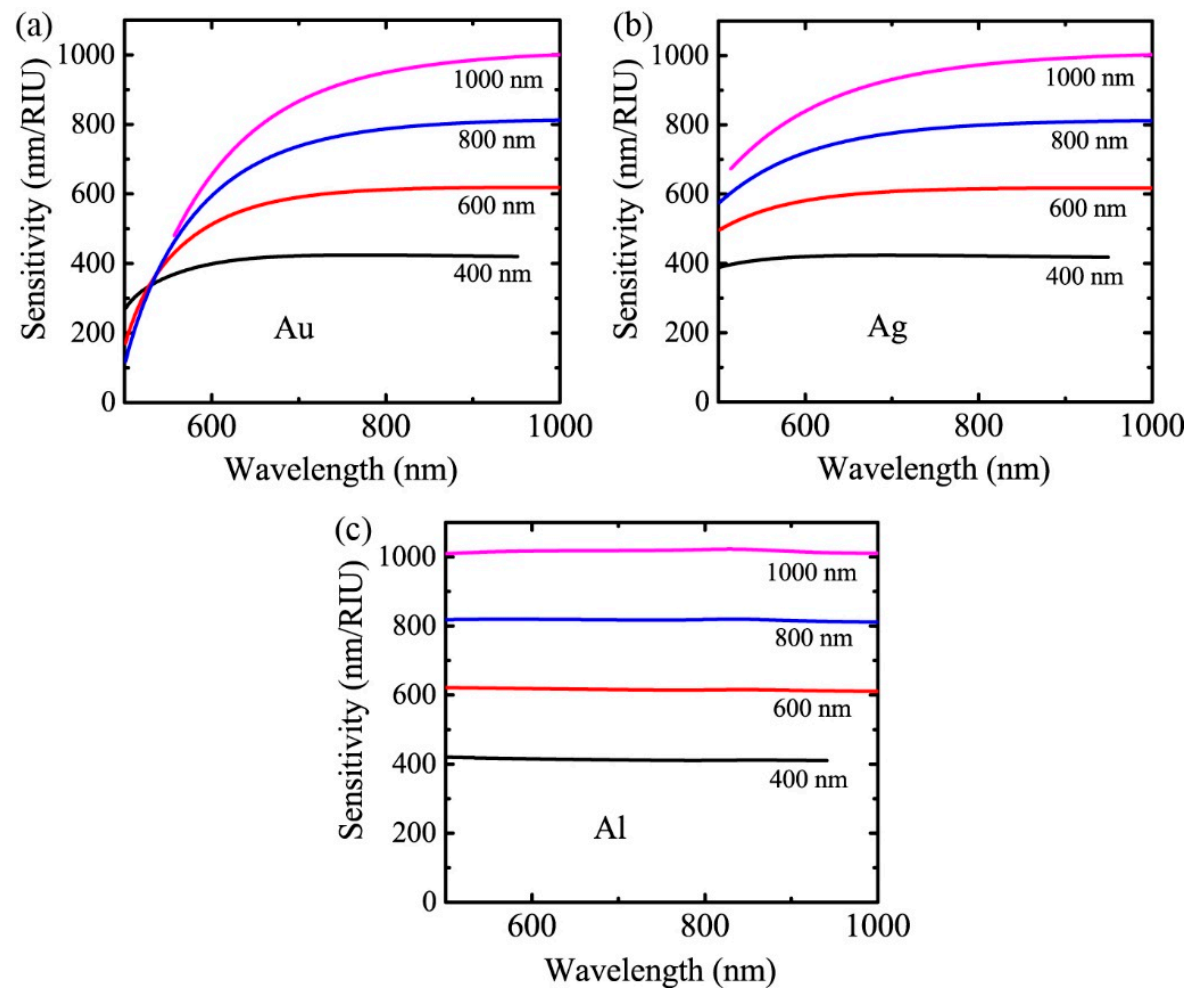

Figure 3. The theoretical sensitivity of the grating-based sensor as a function of the wavelength for four different periods with $n_{a}=1.34, m=1$. The materials for the gratings are (a) Au, (b) $\mathrm{Ag}$, and (c) $\mathrm{Al}$, respectively.

From Equation (5), we can find that $|m| / P$ dominates the behavior of the sensor sensitivity for a given material. At long wavelengths, the numerator in Equation (5) approaches 1 and the second term in the denominator is much smaller than the first term. Therefore, the sensitivity at long wavelengths can be estimated by $P /|m|$. This is very different from the prediction of $\lambda / n_{a}$ calculated in [30]. According to [30], the sensitivity increases with wavelength and is weakly dependent on the diffraction order and period. This leads to the optimization strategy of designing sensors working at the longest possible wavelength and ignoring the choice of grating period and diffraction order. However, for the optical geometry in our case, $S$ increases as the grating period increases and the diffraction order decreases. The wavelength dependence of $S$ varies with the material of the grating. $S$ increases with wavelength and is saturated beyond a certain wavelength for $\mathrm{Au}$ and $\mathrm{Ag}$, while it does not change considerably with wavelength for Al. Thus, the sensors should not only be designed to work at longer wavelengths but also have larger grating periods and work at the \pm 1 st diffraction order.

The large difference between the sensitivity calculated in this paper and in [30] originates from the different models that are considered. In our paper, the light source is outside the analyte channel, while the light source should be inside the analyte channel in [30]. The SPR excitation condition in our case is Equation (4) and that in [30] is Equation (3). Therefore, the expression of the sensitivity derived here is different with that in [30].

Our model has its limits. The incident angle cannot exceed 90 degrees, so Equation (4) should satisfy the constraint of $|\sin \theta| \leq 1$. This constrain limits the grating period that can be applied. For example, at $\lambda=800 \mathrm{~nm}$ and $n_{a}=1.34$, the period of Al grating should be in the range of $340 \mathrm{~nm}$ to $2267 \mathrm{~nm}$.

The dependence of the sensitivity on the refractive index of the analyte in the range of 1 to 2 is depicted in Figure 4. In the calculations, the grating period and the resonant wavelength are assumed 
to be $600 \mathrm{~nm}$ and $800 \mathrm{~nm}$, respectively. As can be seen, $S$ decreases with increasing $n_{a}$ for $\mathrm{Au}$ and $\mathrm{Ag}$, while increases with increasing $n_{a}$ for Al. Since the real parts of the dielectric constants of these metals are negative, both the numerator and the denominator in Equation (5) increase with the refractive index of the sensing medium. The dependence of sensitivity on the refractive index is a result of the competition between the increasing speeds of the numerator and the denominator.

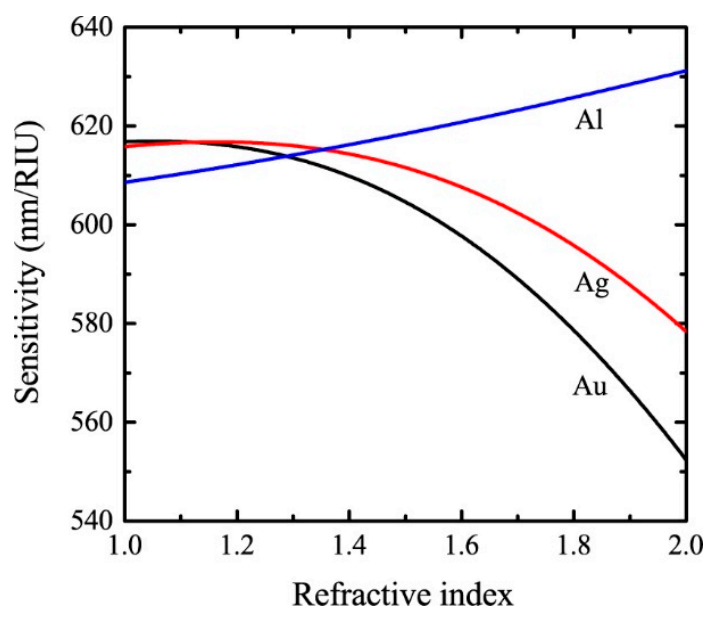

Figure 4. The refractive index dependence of the sensitivity for SPR sensors with a period of $600 \mathrm{~nm}$ and a resonant wavelength of $800 \mathrm{~nm}$.

Our theoretical calculations can be compared with the experimental results in previous literatures. For an Au grating with a period of $320 \mathrm{~nm}$, sensitivities of $425 \mathrm{~nm} / \mathrm{RIU}$ and $360 \mathrm{~nm} / \mathrm{RIU}$ were obtained for $100 \mathrm{~nm}$ and $50 \mathrm{~nm}$ film thickness, respectively [18]. With the same grating period, an $\mathrm{Ag}$ grating-based sensor exhibited sensitivity of $356 \mathrm{~nm} / \mathrm{RIU}$ [17]. Our theoretical analysis is also valid for sensors that measure the enhanced transmission spectra. Sensitivities of $524 \mathrm{~nm} /$ RIU and $600 \mathrm{~nm} /$ RIU were achieved by transmission type sensors based on Au grating with $P=525 \mathrm{~nm}$ and Ag grating with $P=600 \mathrm{~nm}$, respectively [33,34]. According to the above mentioned literatures, we can find that the sensor sensitivities are almost equal to the grating periods $(S \approx P)$, which is in good agreement with our theoretical calculations for sensors working at long wavelengths with $|m|=1$.

\section{Experimental Demonstration}

In order to further demonstrate our theoretical analysis, we experimentally measured the sensitivity of an $\mathrm{Al}$ grating-based sensor over a large wavelength range. The experimental setup is basically the same as that sketched in Figure 1. Detailed information of the setup can be found in our previous paper [35]. The grating was obtained by peeling off the metal layer from a SONY brand Digital Versatile Disc-Recordable (DVD-R) disc manufactured by Sony corporation in Taiwan, China. Here, the grating period and the modulation depth were $720 \mathrm{~nm}$ and $77 \mathrm{~nm}$, respectively, according to the atomic force microscope image. Gratings fabricated by other two companies, namely Unisplendour corporation limited-company (UNIS) and the ANV series of Riteck corporation (ANV), were also measured. The grating periods for UNIS brand DVD-R disc made in Jiangsu, China and ANV brand DVD-R disc made in Taiwan, China were $724 \mathrm{~nm}$ and $727 \mathrm{~nm}$. The modulation depths were $86 \mathrm{~nm}$ and $90 \mathrm{~nm}$. As can be seen, the grating parameters of all the discs were similar. Gratings obtained from DVD-R discs of different manufacturers were proved to excite high quality SPR, and they were suitable to be applied in SPR sensors. In addition, the metal gratings fabricated by our method is stable for a long period of time. SPR response was measured for gratings stored in ambient conditions for more than 2 months. Deionized (DI) water and glucose solutions with concentrations of $5 \%, 10 \%, 15 \%$, and $20 \%$ were used as the analytes. For instance, the reflection spectra measured at the incident angle of $20^{\circ}$ are shown in Figure 5a. As can be seen, there are two absorption dips in the spectra. The deep resonance at the wavelength around $760 \mathrm{~nm}$ was caused by the excitation of 
SPR for the first diffraction order, and the shallow one around $625 \mathrm{~nm}$ was caused by SPR excitation for the second diffraction order. Extracting the SPR wavelengths from the reflection spectra, we can plot the SPR wavelength as a function of the refractive index of the analyte. The results are shown in Figure 5b,c, where the wavelength is linearly dependent on the refractive index. The sensitivities are then obtained by linearly fitting the experimental data. Therefore, for $\theta=20^{\circ}$, the experimental sensitivities are $686.8 \mathrm{~nm} /$ RIU and $358.8 \mathrm{~nm} /$ RIU for $m=1$ and $m=2$, respectively.
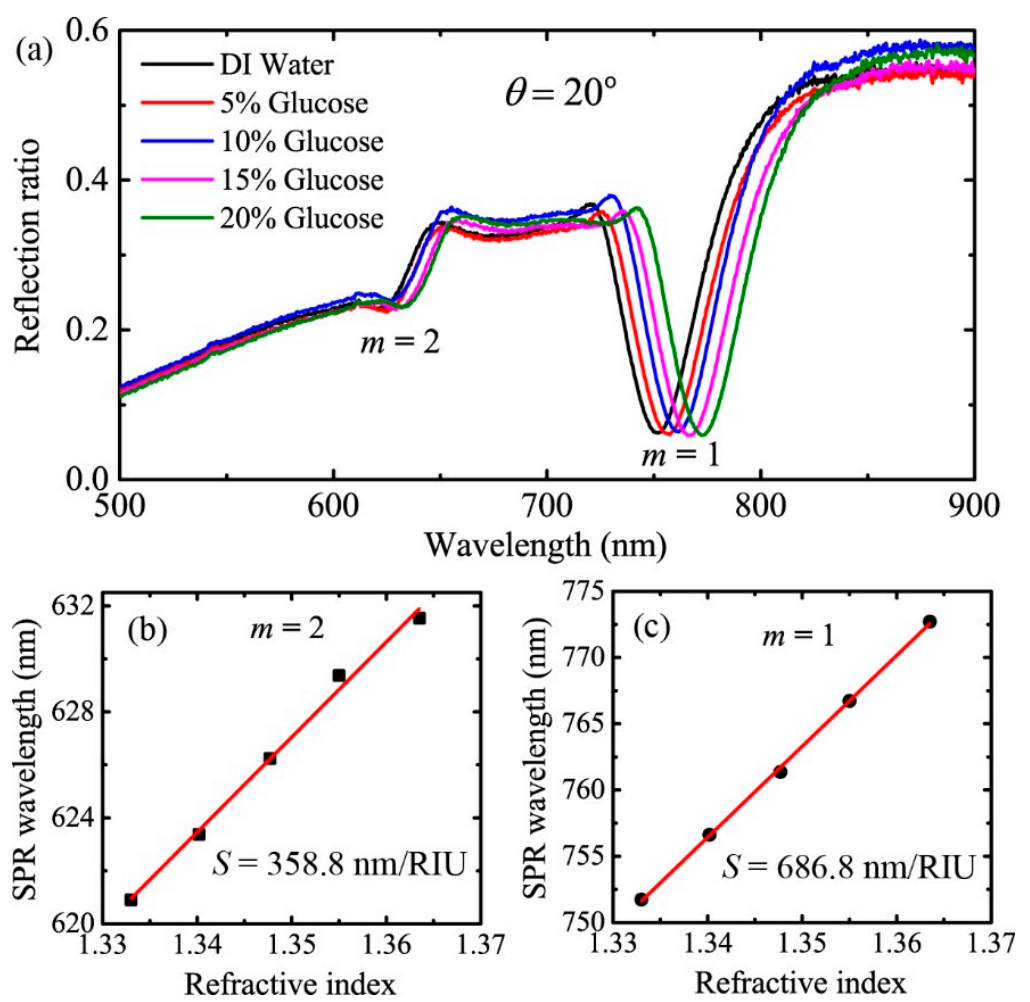

Figure 5. (a) The reflection spectra of the analytes experimentally measured at the incident angle of 20 degrees, $(\mathbf{b}, \mathbf{c})$ are the SPR wavelength as a function of the refractive index for diffraction orders of $m=2$ and $m=1$, respectively. The red lines are linear fits of the experimental data.

Following the above procedure, we obtained the experimental sensitivities for $5 \%$ glucose solution at incident angles of $6^{\circ}, 14^{\circ}, 20^{\circ}, 30^{\circ}, 40^{\circ}$, and $50^{\circ}$. The results are shown as the black circles and red squares in Figure $6 \mathrm{a}$ for the first and second diffraction order, respectively. The theoretical sensitivities under the condition of $P=720 \mathrm{~nm}, n_{a}=1.3402$ are also plotted in Figure $6 \mathrm{a}$ as a comparison. For instance, the theoretical value of the sensitivity for $m=1$ is $737 \mathrm{~nm} / \mathrm{RIU}$ and that for $m=2$ is $372 \mathrm{~nm} / \mathrm{RIU}$ at the resonant wavelength of $700 \mathrm{~nm}$. We can see that the experimental sensitivity does not vary considerably across the analyzing wavelength range for each diffraction order, and the sensitivity for $m=1$ is much higher than that for $m=2$. These characteristics are consistent with the theory. The experimental data are slightly lower than the theory, which may be due to the imperfect alignment of the optical elements and experimental uncertainties. To further confirm the theoretical calculations, it is better to compare sensitivities of sensors with different grating periods. We are working on this and trying to experimentally fabricate metal gratings with various periods.

Other than the sensitivity, the figure of merit $(F O M)$ is another important parameter to characterize sensor performance, which is defined as FOM $=S / F W H M$ (the full width at half maximum of the absorption dip). The experimentally measured FWHM for $5 \%$ glucose solution $\left(n_{a}=1.3402\right)$ with $m=1$ and the corresponding FOM are shown in Figure 6b. The FWHM of the resonance dip decreases with increasing wavelength, which results in increasing FOM. 
The sensitivity is calculated according to the wave vector matching. Since the grating wave vector is only dependent on the period and is independent of the modulation depth, we expect that the modulation depth will not influence the sensitivity, while the modulation depth will certainly influence the absorption line width and absorption depth of the reflection spectra. Therefore, the FOM of the sensor will be influenced. These influences cannot be solved by our analytical expression and can be solved by some numerical models.
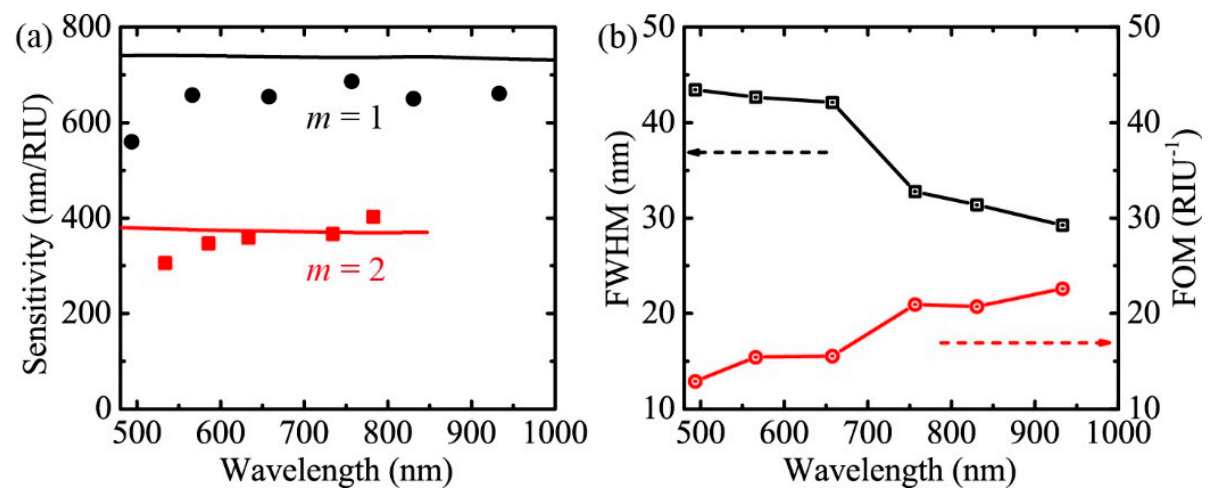

Figure 6. (a) Comparison of the experimental sensitivity and the theoretical sensitivity under the condition of $P=720 \mathrm{~nm}, n_{a}=1.3402$. The black circles and red squares are experimental data. The black and red lines are theoretical data. (b) The FWHM and FOM as a function of the resonant wavelength.

\section{Conclusions}

In conclusion, we have derived analytical expressions for the sensitivity of SPR sensors based on metal gratings with wavelength interrogation. According to the theoretical analysis, the sensitivity increases with increasing wavelength and is saturated beyond a certain wavelength for $\mathrm{Au}$ and $\mathrm{Ag}$ gratings, while it is almost constant for $\mathrm{Al}$ gratings in the wavelength range of 500 to $1000 \mathrm{~nm}$. More importantly, the grating period and the diffraction order dominate the value of sensitivity. Higher sensitivity is possible for SPR sensors with a larger grating period and lower diffraction order. At long wavelengths, a simple expression of $P /|m|$ can be used to estimate the sensor sensitivity. Moreover, we experimentally measured the sensitivity of a SPR sensor based on an Al grating and compared the results with the theory. The wavelength and diffraction order dependence of sensitivity obtained in the experiments are consistent with the theory. The theoretical analysis presented in this paper will guide the optimization of system parameters for SPR sensors. For example, according to the theoretical analysis, a SPR sensor using a metal grating with a period of $1200 \mathrm{~nm}$ can achieve a previously unreported high sensitivity of $1200 \mathrm{~nm} /$ RIU.

Author Contributions: Conceptualization, J.C.; methodology, Y.S.; software, J.C.; validation, Y.K. and W.Q.; formal analysis, J.C.; investigation, J.C.; resources, Y.S.; data curation, Y.S.; writing-original draft preparation, J.C.; writing-review and editing, J.C., Y.S., Y.K., and W.Q.; visualization, Y.K.; supervision, W.Q.; project administration, J.C.; funding acquisition, J.C. and Y.K.

Funding: This research was funded by the National Natural Science Foundation of China (Grant No. 61605067); The Fundamental Research Funds for the Central Universities (Grant No. JUSRP11722, No. JUSRP51721B).

Conflicts of Interest: The authors declare no conflict of interest.

\section{References}

1. Liedberg, B.; Nylander, C.; Lunström, I. Surface plasmon resonance for gas detection and biosensing. Sens. Actuators 1983, 4, 299-304. [CrossRef]

2. Homola, J.; Yee, S.S.; Gauglitz, G. Surface plasmon resonance sensors: Review. Sens. Actuators B 1999, 54, 3-15. [CrossRef] 
3. Wijaya, E.; Lenaerts, C.; Maricot, S.; Hastanin, J.; Habraken, S.; Vilcot, J.P.; Boukherroub, R.; Szunerits, S. Surface plasmon resonance-based biosensors: From the development of different SPR structures to novel surface functionalization strategies. Curr. Opin. Solid State Mater. Sci. 2011, 15, 208-224. [CrossRef]

4. Homola, J. Surface Plasmon Resonance Sensors for Detection of Chemical and Biological Species. Chem. Rev. 2008, 108, 462-493. [CrossRef]

5. Tong, L.; Wei, H.; Zhang, S.; Xu, H. Recent Advances in Plasmonic Sensors. Sensors 2014, 14, $7959-7973$. [CrossRef]

6. Kretschmann, E.; Raether, H. Radiative Decay of Non Radiative Surface Plasmons Excited by Light. Z. Naturforsch. 1968, 23A, 2135-2136. [CrossRef]

7. Otto, A. Excitation of nonradiative surface plasma waves in silver by the method of frustrated total reflection. Z. Phys. A Hadron. Nuclei 1968, 216, 398-410. [CrossRef]

8. Cullen, D.C.; Brown, R.G.; Lowe, C.R. Detection of immuno-complex formation via surface plasmon resonance on gold-coated diffraction gratings. Biosensors 1987, 3, 211-225. [CrossRef]

9. Jory, M.J.; Vukusic, P.S.; Sambles, J.R. Development of a prototype gas sensor using surface plasmon resonance on gratings. Sens. Actuators B Chem. 1994, 17, 203-209. [CrossRef]

10. Bhatia, P.; Gupta, B.D. Surface-plasmon-resonance-based fiber-optic refractive index sensor: Sensitivity enhancement. Appl. Opt. 2011, 50, 2032-2036. [CrossRef]

11. Bhatia, P.; Gupta, B.D. Surface plasmon resonance based fiber optic refractive index sensor utilizing silicon layer: Effect of doping. Opt. Commun. 2013, 286, 171-175. [CrossRef]

12. Singh, S.; Mishra, S.K.; Gupta, B.D. Sensitivity enhancement of a surface plasmon resonance based fibre optic refractive index sensor utilizing an additional layer of oxides. Sens. Actuators A Phys. 2013, 193, 136-140. [CrossRef]

13. Tabassum, R.; Gupta, B.D. Performance Analysis of Bimetallic Layer with Zinc Oxide for SPR-Based Fiber Optic Sensor. J. Lightw. Technol. 2015, 33, 4565-4571. [CrossRef]

14. Tabassum, R.; Gupta, B.D. Influence of Oxide Overlayer on the Performance of a Fiber Optic SPR Sensor with Al/Cu Layers. IEEE J. Sel. Top. Quantum Electron. 2017, 23, 81-88. [CrossRef]

15. Usha, S.P.; Gupta, B.D. Performance analysis of zinc oxide-implemented lossy mode resonance-based optical fiber refractive index sensor utilizing thin film/nanostructure. Appl. Opt. 2017, 56, 5716-5725. [CrossRef]

16. Turker, B.; Guner, H.; Ayas, S.; Ekiz, O.O.; Acar, H.; Guler, M.O.; Dâna, A. Grating coupler integrated photodiodes for plasmon resonance based sensing. Lab. A. Chip. 2011, 11, 282-287. [CrossRef]

17. Guner, H.; Ozgur, E.; Kokturk, G.; Celik, M.; Esen, E.; Topal, A.E.; Ayas, S.; Uludag, Y.; Elbuken, C.; Dana, A. A smartphone based surface plasmon resonance imaging (SPRi) platform for on-site biodetection. Sens. Actuators B Chem. 2017, 239, 571-577.

18. López-Muñoz, G.A.; Estevez, M.C.; Peláez-Gutierrez, E.C.; Homs-Corbera, A.; García-Hernandez, M.C.; Imbaud, J.I.; Lechuga, L.M. A label-free nanostructured plasmonic biosensor based on Blu-ray discs with integrated microfluidics for sensitive biodetection. Biosens. Bioelectron. 2017, 96, 260-267. [CrossRef] [PubMed]

19. Telezhnikova, O.; Homola, J. New approach to spectroscopy of surface plasmons. Opt. Lett. 2006, 31, 3339-3341. [CrossRef] [PubMed]

20. Vala, M.; Chadt, K.; Piliarik, M.; Homola, J. High-performance compact SPR sensor for multi-analyte sensing. Sens. Actuators B Chem. 2010, 148, 544-549. [CrossRef]

21. Yoshiharu, A.; Tetsuo, K.; Kiyoshi, M.; Isao, S. Electrically detectable surface plasmon resonance sensor by combining a gold grating and a silicon photodiode. Appl. Phys. Express 2018, 11, 022001.

22. Shalabney, A.; Abdulhalim, I. Sensitivity-enhancement methods for surface plasmon sensors. Laser Photonics Rev. 2011, 5, 571-606. [CrossRef]

23. Yoon, K.H.; Shuler, M.L.; Kim, S.J. Design optimization of nano-grating surface plasmon resonance sensors. Opt. Express 2006, 14, 4842-4849. [CrossRef]

24. Alleyne, C.J.; Kirk, A.G.; McPhedran, R.C.; Nicorovici, N.-A.P.; Maystre, D. Enhanced SPR sensitivity using periodic metallic structures. Opt. Express 2007, 15, 8163-8169. [CrossRef]

25. Piliarik, M.; Homola, J. Surface plasmon resonance (SPR) sensors: Approaching their limits? Opt. Express 2009, 17, 16505-16517. [CrossRef] [PubMed]

26. Lee, K.-L.; Wei, P.-K. Optimization of periodic gold nanostructures for intensity-sensitive detection. Appl. Phys. Lett. 2011, 99, 083108. [CrossRef] 
27. Rossi, S.; Gazzola, E.; Capaldo, P.; Borile, G.; Romanato, F. Grating-Coupled Surface Plasmon Resonance (GC-SPR) Optimization for Phase-Interrogation Biosensing in a Microfluidic Chamber. Sensors 2018, $18,1621$. [CrossRef]

28. Chen, Z.; Liu, L.; He, Y.; Ma, H. Resolution enhancement of surface plasmon resonance sensors with spectral interrogation: Resonant wavelength considerations. Appl. Opt. 2016, 55, 884-891. [CrossRef]

29. Homola, J. On the sensitivity of surface plasmon resonance sensors with spectral interrogation. Sens. Actuators B Chem. 1997, 41, 207-211. [CrossRef]

30. Homola, J.; Koudela, I.; Yee, S.S. Surface plasmon resonance sensors based on diffraction gratings and prism couplers: Sensitivity comparison. Sens. Actuators B Chem. 1999, 54, 16-24. [CrossRef]

31. Rakić, A.D.; Djurišić, A.B.; Elazar, J.M.; Majewski, M.L. Optical properties of metallic films for vertical-cavity optoelectronic devices. Appl. Opt. 1998, 37, 5271-5283. [CrossRef] [PubMed]

32. Ung, B.; Sheng, Y. Interference of surface waves in a metallic nanoslit. Opt. Express 2007, 15, 1182-1190. [CrossRef] [PubMed]

33. Iqbal, T.; Afsheen, S. One Dimensional Plasmonic Grating: High Sensitive Biosensor. Plasmonics 2017, 12, 19-25. [CrossRef]

34. Xiao, B.; Pradhan, S.K.; Santiago, K.C.; Rutherford, G.N.; Pradhan, A.K. Topographically Engineered Large Scale Nanostructures for Plasmonic Biosensing. Sci. Rep. 2016, 6, 24385. [CrossRef]

35. Sun, Y.; Sun, S.; Wu, M.; Gao, S.; Cao, J. Refractive index sensing using the metal layer in DVD-R discs. RSC Adv. 2018, 8, 27423-27428. [CrossRef]

(C) 2019 by the authors. Licensee MDPI, Basel, Switzerland. This article is an open access article distributed under the terms and conditions of the Creative Commons Attribution (CC BY) license (http://creativecommons.org/licenses/by/4.0/). 